Chinese Journal of Organic Chemistry

\title{
硒化合物苂光传感器研究进展
}

\author{
张继东*, $a$ 詹 妍 ${ }^{a}$ 李胡月雯 $a$ 齐 怡 ${ }^{a}$ 王瑞鹏 $a$ 孟莉 $b$ \\ ( ${ }^{a}$ 安康学院化学化工学院 陕西省富硒食品质量监督检验中心 新型材料研究中心 陕西安康 725000) \\ $\left({ }^{b}\right.$ 中国富硒产业研究院 农业农村部富硒产品开发与质量控制重点实验室 陕西安康 725000)
}

\begin{abstract}
摘要 硒(Se)在生命体内是一种重要的微量元素, 含有硒的蛋白质在哺乳动物体内具有广泛的生物学效应, 如抗氧化、 抗炎和促进产生甲状腺激素等作用. 在过去的几十年里, 含硒物质因其在生物体内的生物活性而引起人们的极大关注, 其主要归因于 $\mathrm{Se}$ 元素的氧化还原及软硬质子性能. 近年来, 随着荧光检测技术的发展, 大量硒化合物荧光传感器被开 发出来. 主要综述了含硒物质如硒代半胱氨酸 $(\mathrm{Sec})$ 、硒化氢、硒醇和 $\mathrm{Se}(\mathrm{IV})$ 等苂光传感器的研究进展, 并对其研究前 景进行了展望.
\end{abstract}

关键词 硒化合物; 苂光传感器; 生物成像; 苂光检测; 纳米颗粒

\section{Recent Progress in Fluorescent Chemosensors for Selenium Compounds}

\author{
Zhang, Jidong ${ }^{*, a} \quad$ Zhan, Yan $^{a} \quad$ Li-Hu, Yuewen ${ }^{a} \quad$ Qi, Yi $^{a} \quad$ Wang, Ruipeng ${ }^{a} \quad$ Meng, $\mathrm{Li}^{b}$ \\ ( ${ }^{a}$ Quality Supervision and Inspection Centre of Se-Enriched Food of Shaanxi Province, Research Centre of New Materials, \\ School of Chemistry \& Chemical Engineering, Ankang Univerisity, Ankang, Shaanxi 725000) \\ $\left({ }^{b}\right.$ Key Laboratory of Se-Enriched Products Development and Quality Control of Ministry of Agriculture, Se-Enriched Products \\ Research Institute of China, Ankang, Shaanxi 725000)
}

\begin{abstract}
Selenium (Se) is an important trace element in life and has been associated with many diseases. Protein containing selenium has a wide range of biological effects, such as antioxidant, anti-inflammatory and promotes the production of thyroid hormone action. In the past few decades, selenium has attracted great attention because of its important role in biology, which is mainly attributed to the redox of Se elements and the properties of hard and soft protons. In recent years, with the development of chemical simulation technology, a large number of selenium-related fluorescent chemosensors have been developed to monitor physiological and pathological processes. Fluorescent chemosensors containing selenium materials, such as selenocysteine (Sec), hydrogen selenide and Se(IV) are reviewed. The development tendency of the sensing Se compounds is prospected.
\end{abstract}

Keywords Se compound; fluorescent sensor; bioimaging; fluorescent detection; nanoparticle

1817 年, 硒元素首次被瑞典化学家 Jöns Jacob Berzelius 发现, 并被命名希腊月亮女神 ${ }^{[1]}$. 作为氧族元 素之一, 其在自然界以六种稳定同位素和多种不同性质 的放射性核素存在. 在生物学方面, 硒元素长期以来被 认为是毒药, 甚至是致癌物质. 然而, Schwarz 和 Foltz 两人更正了这种观点 ${ }^{[2]}$. 后来, 他们研究发现, 含硒的 物质可以防止大鼠肝坏死, 可以作为细菌、哺乳动物和
鸟类的微量营养素. 在近十年来, 硒元素的生物学方面 的研究得到了迅速发展, 硒元素以其多样的形式被认为 是生命系统中必需的微量元素 ${ }^{[3]}$. 在人体中, 硒的生物 功能是通过以 25 种硒代半胱氨酸为活性中心的硒蛋白 作用来实现 ${ }^{[4]}$. 这些蛋白质与人类和其它哺乳动物健康 有关，如降低癌症发病率、预防心血管疾病、预防阿尔 兹海默症、治疗特定的肌肉疾病以及延缓艾滋病毒阳性

\footnotetext{
* Corresponding author. E-mail: akuzjd@aku.edu.cn

Received February 19, 2020; revised April 9, 2020; published online April 23, 2020.

Project supported by the Youth Foundation of Shaanxi Provincial Science \& Technology Department (No. 2019JQ-504), the Key Laboratory of Se-Enriched Products Development and Quality Control, Ministry of Agriculture (No. Se-2018B02), the Doctor's Initial Funding of Ankang University (No. 2018AYQDZR06) and the National Undergraduate Training Program for Innovation and Entrepreneurship (Nos. 201911397007, 201911397016). 陕西省青年科学基金(No. 2019JQ-504)、农业部富硒产品开发国家地方联合工程实验室开放课题(No. Se-2018B02)、安康学院博士科研启动基金(No. 2018AYQDZR06)和国家级大学生创新创业训练计划(Nos. 201911397007, 201911397016)资助项目.
} 
患者发病的时间等 ${ }^{[5-6]}$. 科学家们已经证实硒在不同的 生理和病理过程中发挥着重要作用, 低水平硒会加剧疾 病的致死率、免疫功能低下和认知能力下降的风险, 而 高水平的硒则具有抗病毒作用. 因此, 硒元素受到化学 及生命科学研究工作者的高度关注 ${ }^{[7-10]}$.

荣光分析法是检测分析物方法中最简单、廉价和快 速的方法之一. 苂光传感器由于其具有高灵敏度和高选 择性, 特别是可以用于样品的体内原位成像检测, 目前 已在许多领域得到广泛应用. 近年来, 一些能够监测生 理和病理过程的苂光传感器被报道 ${ }^{[11-18]}$. 本文主要总结 了近年来硒化合物荧光传感器的研究进展, 并对该领域 的研究进行了总结和展望.

\section{1 硒代半胱氨酸(Sec)荧光传感器}

硒代半胱氨酸 $(\mathrm{Sec})$ 是半胱氨酸 $(\mathrm{Cys})$ 的类似物, 是 基因正常编码的第 21 个氨基酸 ${ }^{[19]}$. 在生物体系中, 硒 代半胱氨酸也是硒元素的主要存在形态, 在亚硒酸盐代 谢中起重要作用. 硒代半胱氨酸也是唯一含有准金属元 素的氨基酸, 与各种人类疾病密切相关, 在癌症的预防 和治疗中起重要作用. 此外, 研究 Sec 的重要生理功能 并清晰地阐明硒的抗癌机制, 需要找到具有生物相容 性, 同时能够通过可靠且快速地检测 Sec 的化学传感器 来监测活细胞或体内 $\mathrm{Sec}$ 的方法.

2006 年, Maeda 等 ${ }^{[20]}$ 首次设计合成了一种硫醇的苂 光传感器 $\mathbf{1}$, 该荧光传感器可用于胆碱酯酶的苂光分析, 且该传感器的荧光反应是硫醇阴离子通过芳香亲核取 代脱保护而发生的. 上述作用机制表明, 该传感器作为 硫醇传感器对于硫醇的解离程度是起重要作用的. 此 外, 该传感器还具有探测硫醇或硒醇的能力, 仅通过将 培养基的 $\mathrm{pH}$ 值从 7.4 改为 5.8 , 就可作为硒醇的传感器 (图 1). 进一步研究发现, 通过实验可以证实微酸性介质
的存在不影响该探针作为硒醇传感器的应用。该结构是 第一例报道的能够检测硒醇的荧光传感器. 该传感器的 相关实验提供了一种可用于鉴定和测量具有独特化学 和生物学特性的硒蛋白的工具.
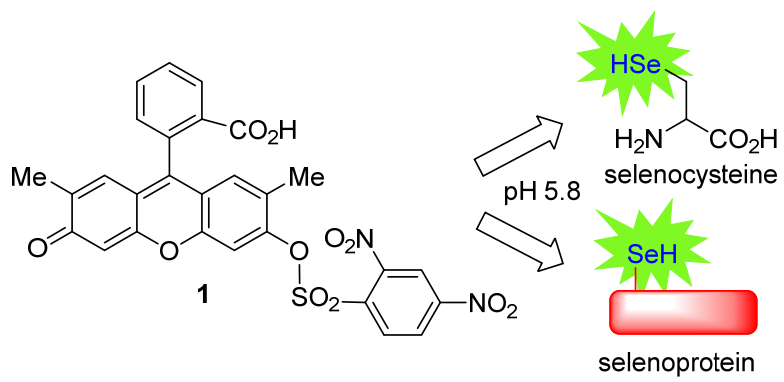

图 1 苂光传感器 $\mathbf{1}$ 的化学结构和区分硒代半胱氨酸和硒蛋白 示意图

Figure 1 The chemical structure fluorescent sensors 1 and the scheme of discrimination between selenocysteine and selenoprotein

2017 年，袁林等 ${ }^{[21]}$ 开发了两种用于特异性检测硒 醇的荧光传感器 2 和 3 (图 2). 在生理 $\mathrm{pH}$ 条件下, 传感 器 2 对硒醇表现出高选择性和灵敏性. 该传感器无毒且 具备超低的背景苂光, 在 Sec 存在下能显示出 380 倍的 苂光信号增强的现象，适用于活细胞中的外源性和内源 性硒醇的荧光检测和成像研究. 除此之外, 该传感器还 被成功应用于脂多糖(LPS)诱导的 HepG2 细胞中硒醇浓 度的可视化检测.

2015 年，房建国等 ${ }^{[22]}$ 基于芳香亲核取代反应报道 了一种选择性 Sec 荧光传感器 4(图 3). 作者经过初步筛 选后，总结了 Sec 选择性识别的构效关系. 研究发现, 在 Sec 存在的情况下, 在中性水溶液中其发射强度增加 100 倍以上，且没有明显的生物硫醇、胺类和醇类的干 扰. 该传感器被成功应用于定量硒酶硫氧还蛋白还原酶

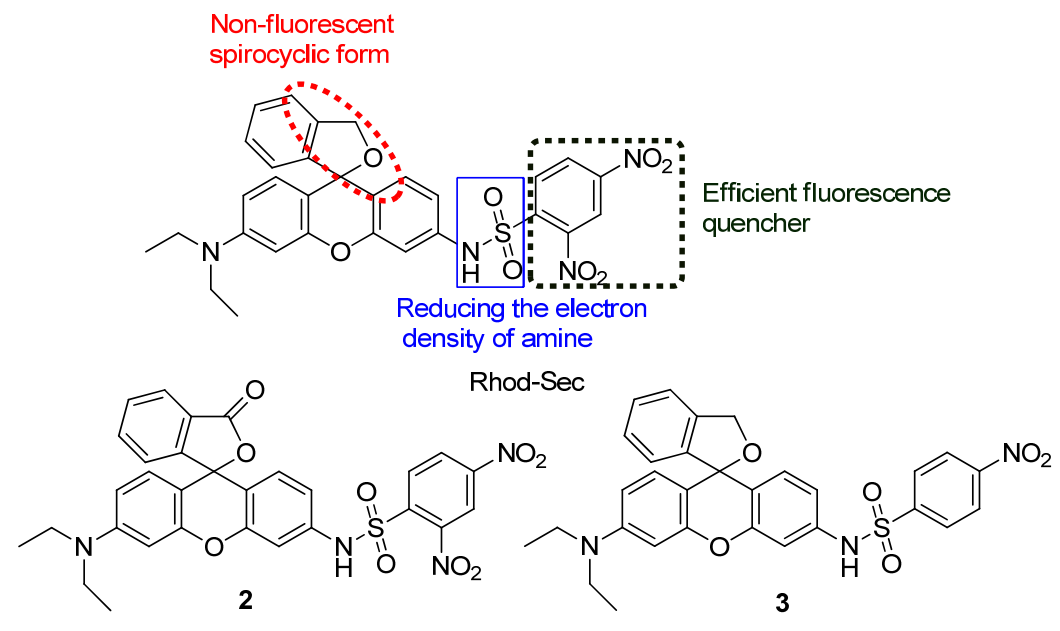

图 2 荧光传感器 2 和 3 的化学结构和设计原理示意图

Figure 2 Chemical structures and design principle of fluorescent sensors 2 and 3 
中的 Sec 含量和活的 HepG2 细胞中内源性 Sec 实时成像. 该研究工作是第一例在生理条件下的硒醇探针. 该研究 工作最大的亮点在于阐明了传感器选择性识别硒醇的 构效关系, 为进一步设计新传感器以及更好地理解 $\mathrm{Sec}$ 和硒蛋白在体内的关键作用提供依据.
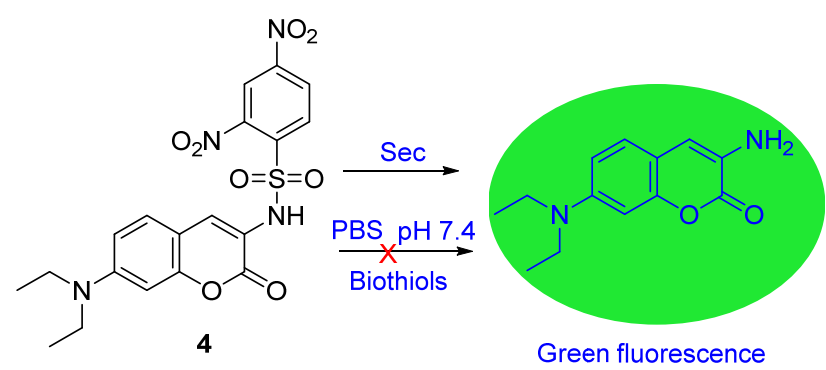

图 3 苂光传感器 4 的化学结构和在中性水溶液中对 Sec 反应 示意图

Figure 3 Chemical structure of fluorescent sensor 4 and its reaction to Sec in neutral aqueous solution

2018 年, 冯国强等 ${ }^{[23]}$ 报道了一种 $\operatorname{Sec}$ 苂光传感器 $\mathbf{5}$, 尽管该结构在生物硫醇检测方面已有报道, 但该研究工 作表明, 只需稍微改变检测条件, 该传感器就可以在水 溶液中快速、高选择性、灵敏地比色和荧光检测 Sec(图 4). 该传感器的检出限为 $47 \mathrm{nmol} / \mathrm{L}$ 左右, 线性范围为
$0 \sim 80 \mu \mathrm{mol} / \mathrm{L}$. 此外, 该传感器具有较低的细胞毒性, 并且能够检测血清和活细胞中的 Sec, 可作为一种 Sec 新型分子检测工具.

2016 年，杨光富等 ${ }^{[24]}$ 报道了一种能够识别硒醇分 子结构的苂光传感器 6 (图 5). 该传感器表现出接近零背 景荧光, 与 Sec 反应具有大的荧光发射增强、低检测限 $(18 \mathrm{nmol} / \mathrm{L})$ 和较大的反应速率常数 $\left(2.15 \mathrm{~min}^{-1}\right)$. 此外, 该传感器能够用于细胞内和组织中含硒的分子(如 Sec 和硒蛋白)的高度特异性和灵敏性检测. 上述结果表明, 该传感器可以作为活细胞和活体内硒醇感应的检测工 具, 从而进行含有内源性硒醇的分子检测. 该结构有望 用于 Sec 相关生物学功能研究和 Se 缺乏或过量相关地 区人类疾病的临床诊断和治疗. 在该课题组 ${ }^{[25]}$ 之前的 研究工作中发现, 香豆素环 7 位上的氮原子内部扭曲受 阻可以显著提高游离荧光团的量子产率.

2017 年, 冯国强等 ${ }^{[26]}$ 报道了一种比色和近红外检 测 Sec 的苂光传感器 7(图 6). 该传感器可用于检测生物 体内的 Sec. 该传感器在较为温和的条件以及较宽 $\mathrm{pH}$ 范 围的水溶液, 对 Sec 具有高灵敏性和高选择性. 该传感 器具有较大的斯托克斯位移 $(192 \mathrm{~nm})$ 和较低的检出限 $(62 \mathrm{nmol} / \mathrm{L})$. 其检测速度快、选择性高且灵敏度好, 并 具有显著的近红外荧光发射信号. 除此之外，该传感

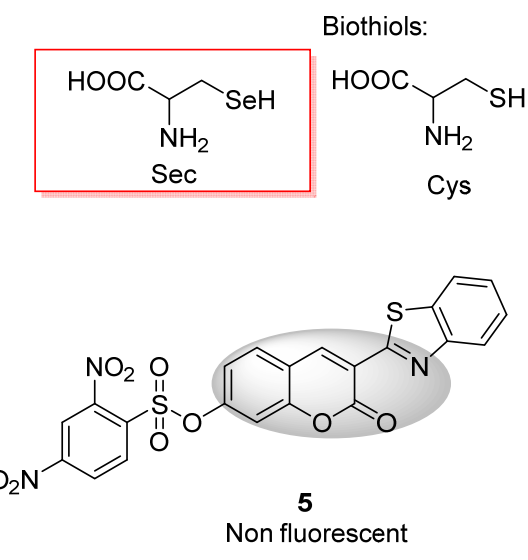<smiles>NC(CCC(=O)NC(CS)C(=O)NCC(=O)O)C(=O)O</smiles>
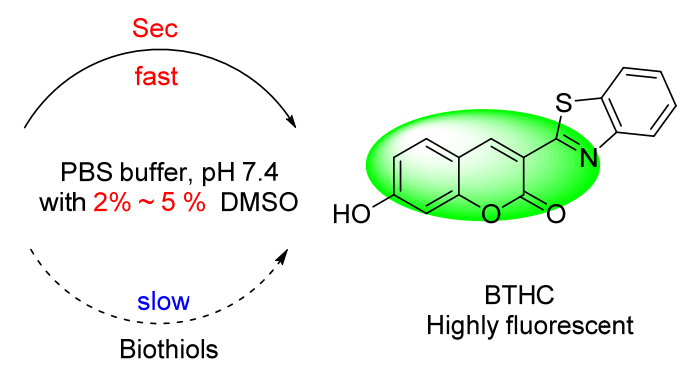

图 4 荧光探针 $\mathbf{5}$ 的化学结构和检测 $\operatorname{Sec}$ 的机理示意图

Figure 4 Chemical structure of fluorescent sensor $\mathbf{5}$ and the mechanism of detecting Sec

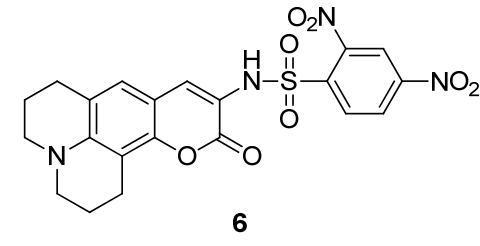

No fluorescence

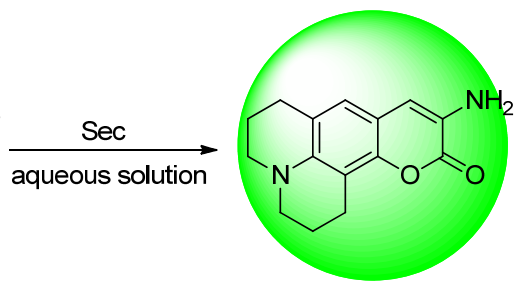

Strong fluorescence

图 5 传感器 6 的化学结构和检测 $\operatorname{Sec}$ 的机理示意图

Figure 5 Chemical structure of sensor 6 and the mechanism of Sec detection 


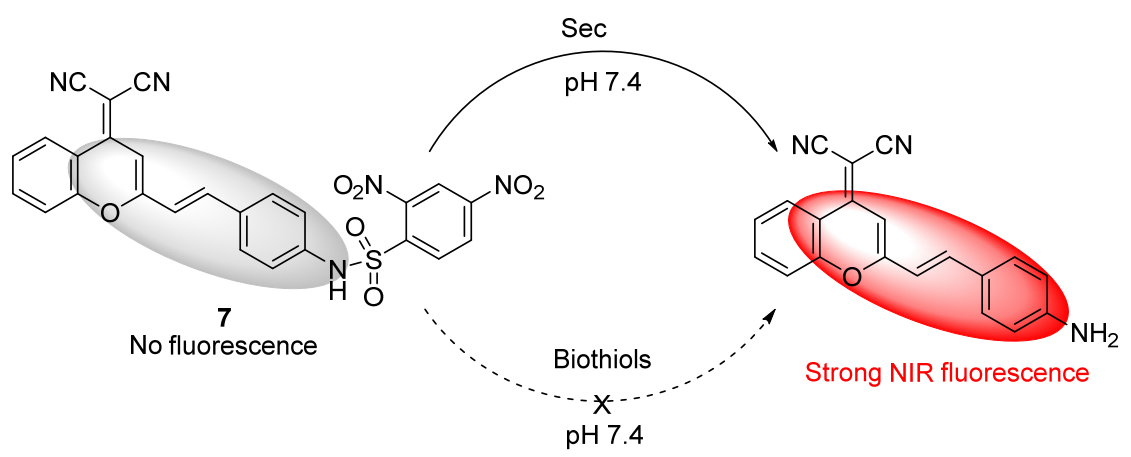

图 6 荧光传感器 7 的化学结构和其可见区对 $\operatorname{Sec}$ 的苂光响应示意图

Figure 6 Chemical structure of fluorescent sensor 7 and the fluorescence response of its visible region to Sec

器还具有较低的细胞毒性, 可以用于检测血清、活细胞 和动物体中的 Sec.

同年，该课题组 ${ }^{[27]}$ 报道了一种 Sec 比色和近红外的 苂光传感器 8(图 7). 该传感器同样表现出显著的斯托克 斯位移 $(146 \mathrm{~nm})$, 并在检测 Sec 时显示出快速、高选择 性和高灵敏性的特点, 同时还具有明显的颜色变化, 并 出现近红外苂光信号. 此外, 该传感器的线性范围是 $0.2 \sim 80 \mu \mathrm{mol} / \mathrm{L}$, 其检测限为 $62 \mathrm{nmol} / \mathrm{L}$. 通过研究进一 步证明, 该传感器可用于检测活细胞中的外源性和内源 性 Sec, 并可用于苂光成像研究. 该研究结果为未来 Sec 荧光传感器设计提供了一种新的设计思路. 另外, 该工 作最大的特点是能够比色检测 Sec.
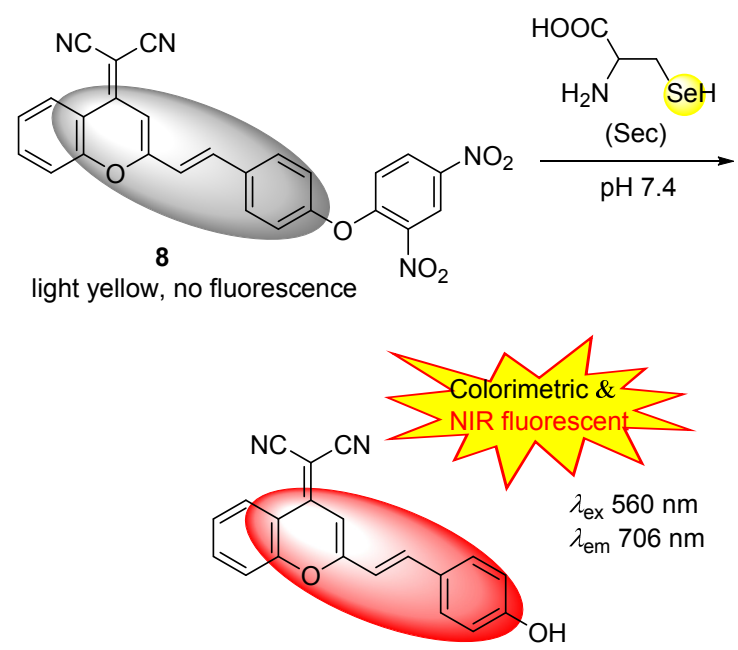

purplish red, strong NIR fluorescence

图 7 荧光传感器 $\mathbf{8}$ 的化学结构以及对硒半胱氨酸的比色和近 红外苂光检测示意图

Figure 7 Chemical structure of fluorescent sensor 8 and the scheme of colorimetric and near-infrared fluorescence detection of selenium cysteine

Liu 等 ${ }^{[28]}$ 以 2,4-二硝基苯磺酸官能团为识别基团, 设计合成了一种以四氢萗-呫吨染料为基础的新型苂光 传感器 9(图 8). 该传感器能够在 $2 \mathrm{~min}$ 内快速检测出
$\mathrm{Sec}$, 并且在 $624 \mathrm{~nm}$ 处有较大的苂光发射增强(增强 124 倍). 其对 Sec 具有很强的选择性, 灵敏度高, 检出限可 以低至 $17 \mathrm{nmol} / \mathrm{L}$. 此外，该结构还被用于 $\mathrm{Sec}$ 的荧光成 像研究, 在活细胞中具有良好的生物相容性.

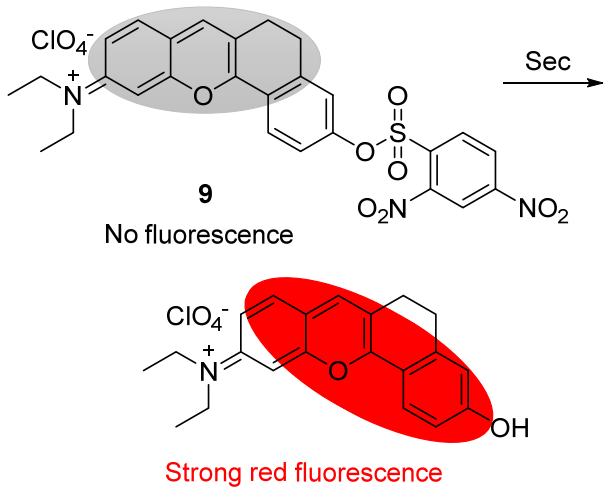

图 8 传感器 9 的化学结构以及与 $\operatorname{Sec}$ 反应的较大苂光增强示 意图

Figure 8 Chemical structure of sensor 9 and larger fluorescence enhancement of Sec reaction

2017 年, 张小玲等 ${ }^{[29]}$ 为了评估 $\mathrm{Sec}$ 的浓度和分布, 设计合成了基于分子内电荷转移(ICT)效应的比率型荧 光传感器 10, 并将其应用于选择性区分活细胞基质中 的内源性 Sec(图 9). 该传感器由基于 ICT 荧光报告的 Nap-OH 支架和作为 Sec-active 触发器单元组成. 研究发 现, 该传感器可在生理条件下对 Sec 表现出特异选择性 反应，并在 $1 \mathrm{~min}$ 内显示出较为明显的比率和红移的荧 光信号. 此外, 该传感器对 Sec 快速响应实现比率性检 测, 能够实时监控细胞内 $\operatorname{Sec}$ 的生成, 同时可用于对活 细胞的外源性和内源性 Sec 成像研究. 后续研究发现, 传感器 10 在生理条件下, 对 Sec 选择性的响应能力超过 生物硫醇、胺或活性氧物质.

2015 年, 唐波等 ${ }^{[30]}$ 通过将苯并硒唑结构引入到半 花菁染料结构中, 合成了一种新型的苂光传感器 11(图 10). 该传感器首次实现了对 $\operatorname{Sec}$ 的高灵敏性和选择性检 


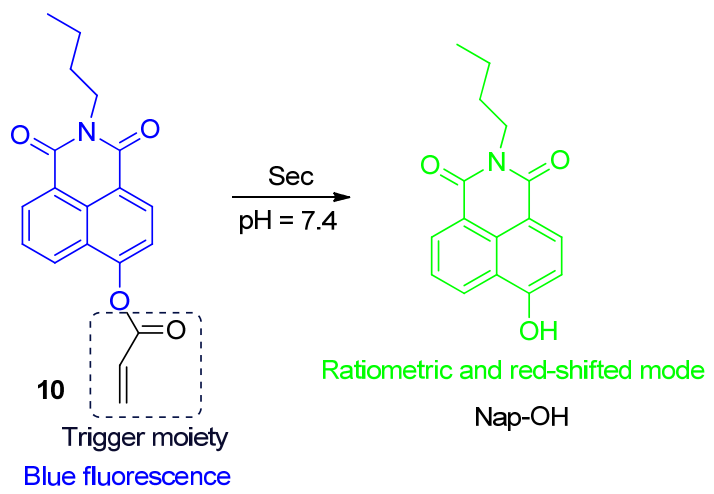

图9 比率荧光传感器 $\mathbf{1 0}$ 的化学结构以及在生物条件下对 $\mathrm{Sec}$ 的特异选择性反应示意图

Figure 9 Chemical structure of the ratio fluorescent sensor 10 and the scheme of specific selective reaction to Sec under biological condition
测，其在生理条件下的检测性能超过其它生物物种，如 硫醇、各种基本离子和氨基酸. 为了研究 $\mathrm{Na}_{2} \mathrm{SeO}_{3}$ 的抗 癌机理, 作者第一次将 2,1,3-苯并硒二唑(BS)部分结合 到半花青染料中. 该传感器被成功应用于细胞成像和活 体内 Sec 浓度变化的监测. 通过细胞周期和线粒体膜电 位分析发现, 经过 $\mathrm{Na}_{2} \mathrm{SeO}_{3}$ 诱导的 $\mathrm{HepG} 2$ 细胞, 其细胞 调亡与硒醇水平密切相关. 该研究结果有利于人们理解 硒化合物在癌症治疗和预防中机理. 该工作是第一例对 活细胞和活体内的硒(RSeH)进行成像研究.

2018 年, 于法标等 ${ }^{[31]}$ 设计合成了一种线粒体靶向 近红外比率型苂光传感器 $\mathbf{1 2}$, 用于定量评估 $\operatorname{Sec}$ 对二硫 化碳 $\left(\mathrm{CS}_{2}\right)$ 诱导的细胞和肝损伤的保护效果(图 11). $\mathrm{CS}_{2}$ 是常见的工业溶剂, 可抑制氧化还原酶和诱导氧化应 激. 该苂光传感器由以下部分组成: 近红外七甲基菁结

(a)

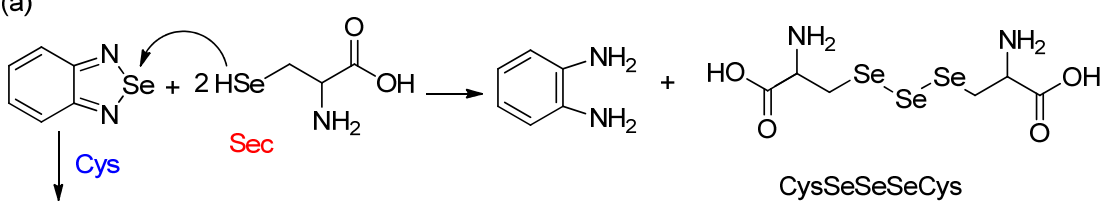
No reaction

(b)

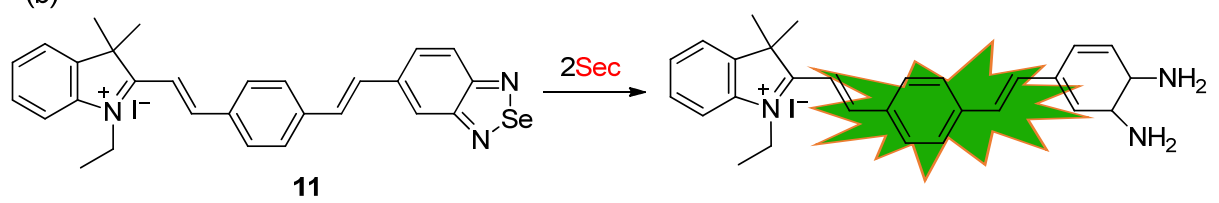

图 10 苂光传感器 11 的化学结构和设计策略

Figure 10 Chemical structure and the design strategy of fluorescent sensor 11<smiles></smiles>

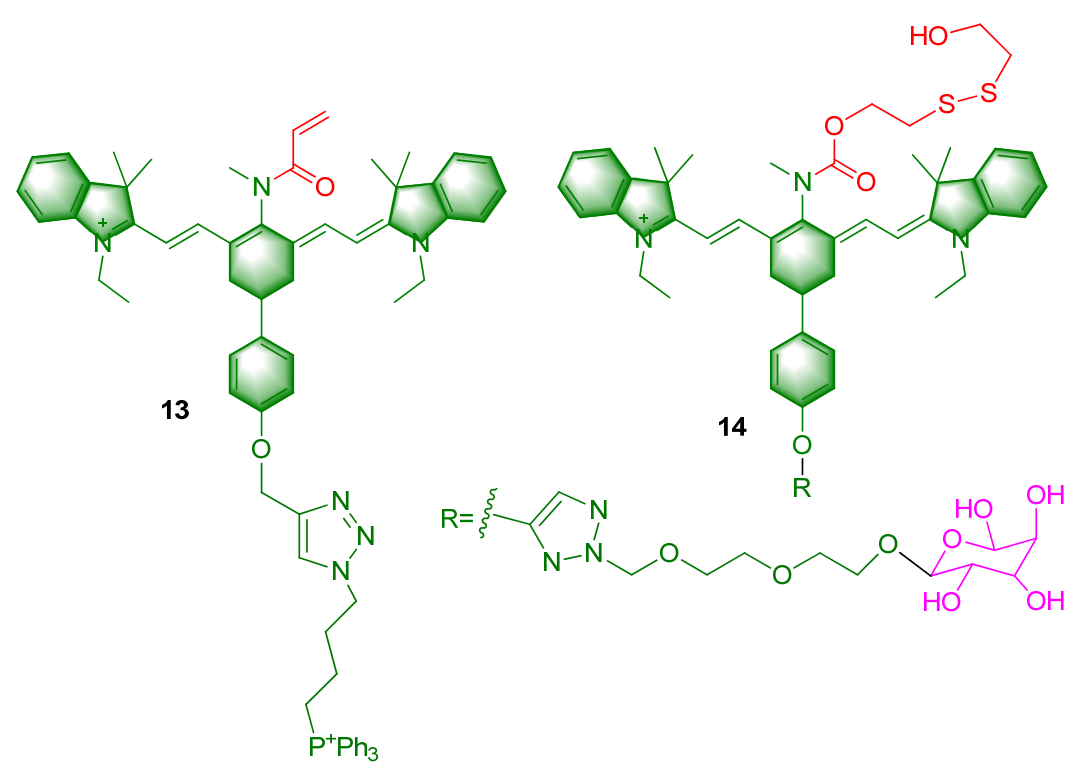

图 11 苂光传感器 12 14 的化学结构

Figure 11 Chemical structures of fluorescent sensors 12 14 
构作为荧光发光基团, 2,4-二硝基苯磺酰胺作为待测物 响应单元, $\mathrm{PPh}^{3+}$ 作为线粒体靶向官能团. 该传感器可在 $4 \mathrm{~min}$ 内快速对 Sec 响应, 不受其它金属离子和阴离子 干扰. 此外, 该传感器可用于选择性测定 BRL3A、 RH-35、HL-7702、HepG2 等细胞系中的内源性 Sec 浓 度. 该传感器有望应用于监测 $\mathrm{CS}_{2}$ 诱导的线粒体中的 $\mathrm{Sec}$ 浓度变化. 该工作最大的亮点是建立了 $\mathrm{CS}_{2}$ 诱导的 急慢性肝炎小鼠模型, 以研究 $\mathrm{CS}_{2}$ 和 $\mathrm{Sec}$ 在肝脏中的细 胞保护作用. 研究发现, Sec 的外源性补充可有效防御 $\mathrm{CS}_{2}$ 引起肝脏损害. 肝脏中 $\mathrm{Sec}$ 浓度的实时成像被用于 评估 CS2 中毒导致的肝损伤程度. 该研究结果有望使该 结构成为临床准确诊断和治疗潜在的候选药物. 2020 年, 他们 ${ }^{[33]}$ 在此结构的基础上设计合成了一种比率型 近红外荧光探针 13, 该探针可以用于分析 $\operatorname{Sec}$ 在细胞和 小鼠甲状腺疾病模型中的变化和作用. 在 2017 年, 该课 题组 ${ }^{[32]}$ 还报道了一种以(2-羟乙基)二硫化物为响应单元 和 $d$-半乳糖结构为肝脏靶向单元的比率型 $\operatorname{Sec}$ 荧光探针 14.

2018 年, 杨小峰等 ${ }^{[34}$ 设计合成了一种基于半花菁 结构的 Sec 荧光传感器 13, 席夫碱结构和半花菁结构的 互补效应导致了其独特的 OFF-ON 荧光转变(图 12). 传 感器 13 对 Sec 的荧光响应是通过 Sec 选择性地移除强 吸电子的 2,4-二硝基苯磺酸基团实现. 传感器亚胺氮的 $\mathrm{p} K_{\mathrm{a}}$ 值从 6.40 转变到 9.04. 因此, 该席夫碱结构在生理 $\mathrm{pH}$ 条件下, 大大增加了半花菁染料的荧光强度. 此外, 传感器 13 对 $\operatorname{Sec}$ 表现出良好的选择性和灵敏性, 已成功 应用于活细胞中硒醇的外源性和内源性苂光成像检测。 该结构对含二硫醇的蛋白质(VDP)的选择性比生物硫醇 和其他蛋白质高, 并且能够对活细胞中的内源性 VDP 成像. 该研究结果是首次报道的通过调节亚胺 $\mathrm{pKa}$ 实
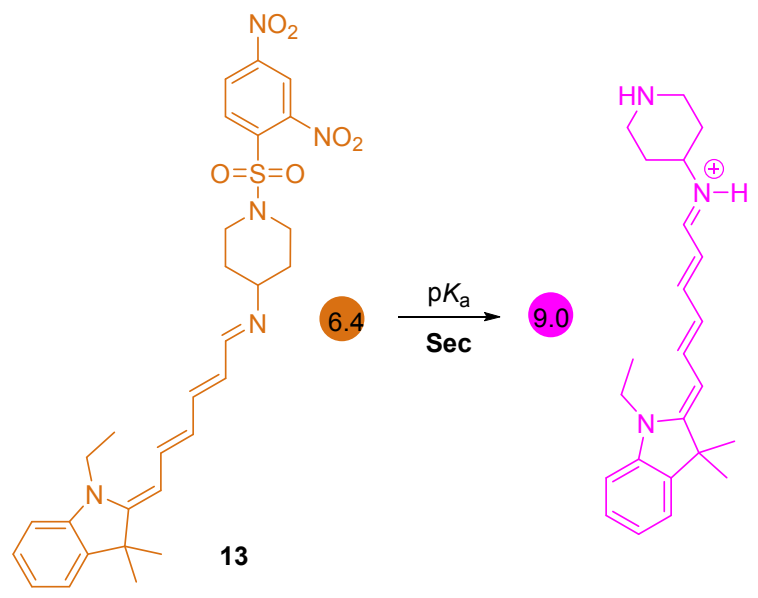

图 12 荧光传感器 13 的化学结构和导致探针亚胺氮 $\mathrm{p} K_{\mathrm{a}}$ 值转 变过程

Figure 12 Chemical structure of fluorescent sensor 13 and the $\mathrm{p} K_{\mathrm{a}}$ value transition process of probe imine nitrogen
现较大位移的靶向蛋白传感体系，其结果有望用于开发 其它新型目标分子苂光传感器.

2015 年, 林伟英等 ${ }^{[35]}$ 设计合成了能够用于特异性 检测硒醇的近红外荧光传感器 $\mathbf{1 4}$, 其在生理 $\mathrm{pH}$ 下表现 出良好的选择性，在硒醇存在下其荧光发射强度增强 65 倍. 该传感器具有良好的生物相容性, 能够快速定量 检测 Sec. 生物性能研究发现该结构不仅适用于活细胞, 也适用于活体动物中硒醇荧光成像研究. 该结构的缺点 在于对硒醇响应的荧光发射增强有限.

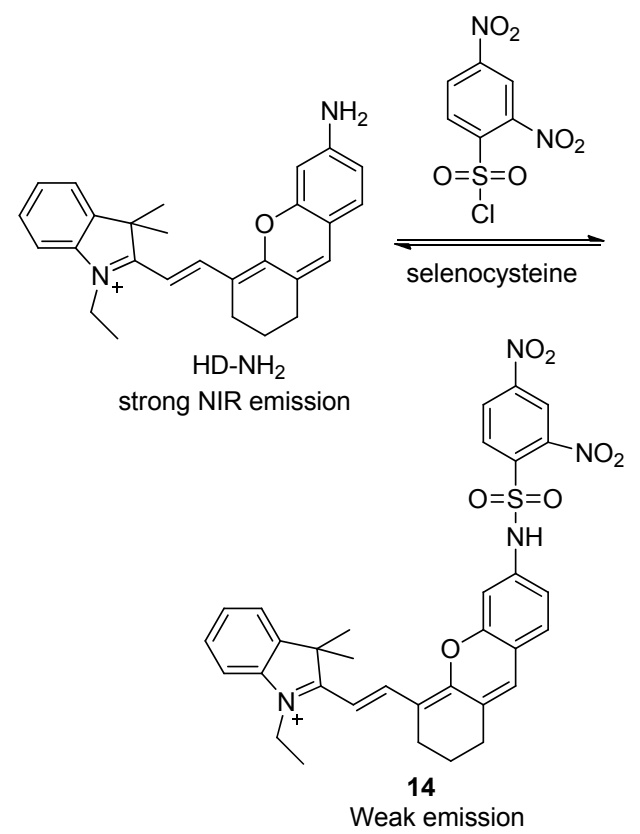

图 13 近红外(NIR)苂光传感器 14 的化学结构和对硒醇的选 择性反应示意图

Figure 13 Chemical structure of near-infrared (NIR) fluorescent sensor $\mathbf{1 4}$ and the scheme of selective reaction to selenium alcohol

最近，印晓星等 ${ }^{[36]}$ 开发了一种新的近红外苂光传 感器 15 , 用于测定活细胞和体内的内源性 Sec. 传感器 与 $\mathrm{Sec}$ 反应时, 表现出较大的苂光增强(136 倍)和较大的 Stokes 位移 $(195 \mathrm{~nm}$ ). 传感器具有灵敏度高(检测限 10 $\mathrm{nmol} / \mathrm{L}$ )、选择性好、细胞毒性低等优点，同时能够识别 活细胞中的外源性和内源性 Sec. 该传感器还被成功地 用于外源性和内源性的小鼠体内 Sec 的可视化检测. 值 得注意的是, 反应性近红外传感器首次选择性地检测了 移植到裸鼠活体肿瘤中的内生生成的 Sec. 该研究结果 表明, 所报道的新传感器可以作为一种有效的工具, 监 测体内内源性 Sec 和探索硒的抗癌机制.

2019 年, 李敏勇等 ${ }^{[37]}$ 首次合成了一种生物发光传 感器 16, 其可以作为一种有效的工具来测定活细胞和 活体中的 $\operatorname{Sec}($ 图 15). 该传感器具有灵敏度高(检测限为 


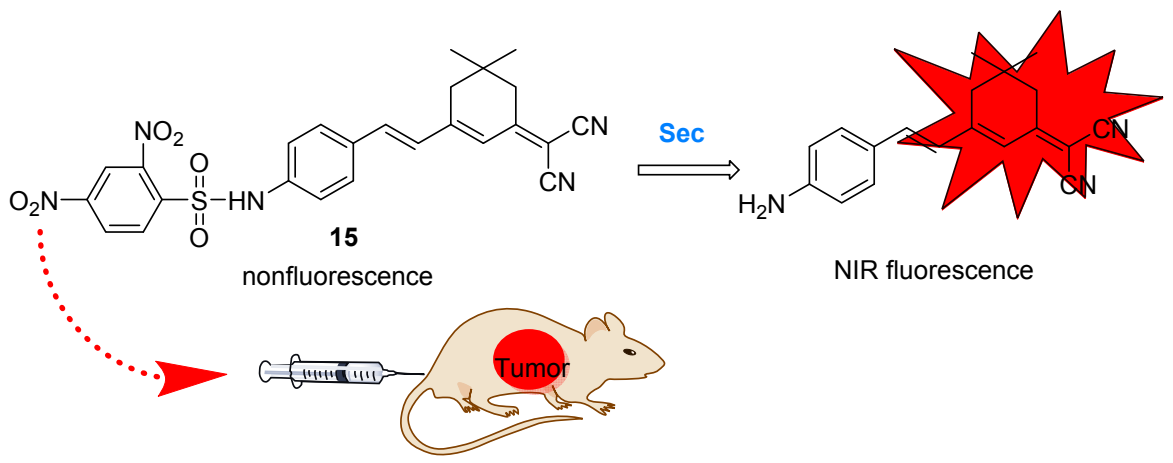

图 14 传感器 $\mathbf{1 5}$ 检测 $\mathrm{Sec}$ 的机理图

Figure 14 Detection mechanism of chemosensor 15 for Sec

$8 \mathrm{nmol} / \mathrm{L}$ )、生物发光增强显著(580 倍)、选择性可靠、 细胞毒性低和信噪比高等优点, 可用于活体细胞和小鼠 中的 Sec 的成像检测. 更重要的是, 其对监测 $\mathrm{Na}_{2} \mathrm{SeO}_{3}$ 刺激的荷瘤小鼠的 Sec 具有很高的灵敏度. 这些研究结 果表明, 该传感器可以作为体内选择性监测 Sec 的有力 工具, 从而为探索硒的生理病理功能和抗癌机制提供了 一条有价值的途径.

刺激响应胶束具有稳定、灵敏和制备方便等优势, 常用的刺激源为 $\mathrm{pH}$ 、温度、光照、氧化还原电位、超 声波、电荷、气体、生物分子和酶 ${ }^{[38-41]}$. 2015 年, 邱仁 华等 ${ }^{[42]}$ 制备了一种 2,4-二硝基苯磺酰基修饰的碳酸盐共 聚物胶束生物传感器 $\mathbf{1 7}$, 在生理 $\mathrm{pH}$ 条件下, 用于 $\mathrm{Sec}$ 选择性成像研究. 研究发现, 捕获阿霉素(DOX)药物的 传感器能够选择性地对 Sec 产生反应, 而很少受到生物 硫醇、胺或醇的干扰(图 16). 该课题组成功地将该传感 器应用于肿瘤组织和 Hela 细胞内源性 Sec 的荧光成像, 同时观察到了阿霉素药物释放过程. 此外, 该传感器具
有疏水分子传递的控制作用. 研究发现, 被捕获的 DOX 药物能够有效释放. 该结果是首次报道的通过胶束实现 Sec 检测和 DOX 药物的释放.

2017 年, 杨小峰等 ${ }^{[43]}$ 报道了一种用量子点(carbon dots, CDs)作为荧光基团的硒醇纳米探针 18(图 17). 该

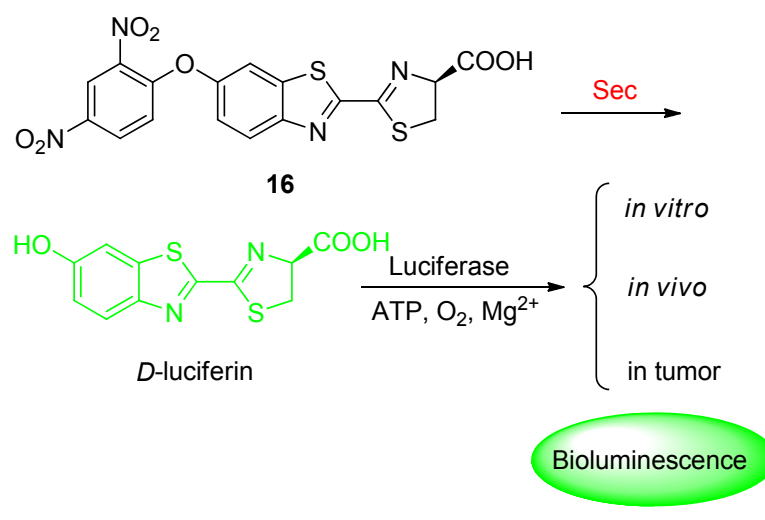

图 15 传感器 16 对 $\operatorname{Sec}$ 的检测机理

Figure 15 Proposed response mechanism of $\mathbf{1 6}$ for Sec

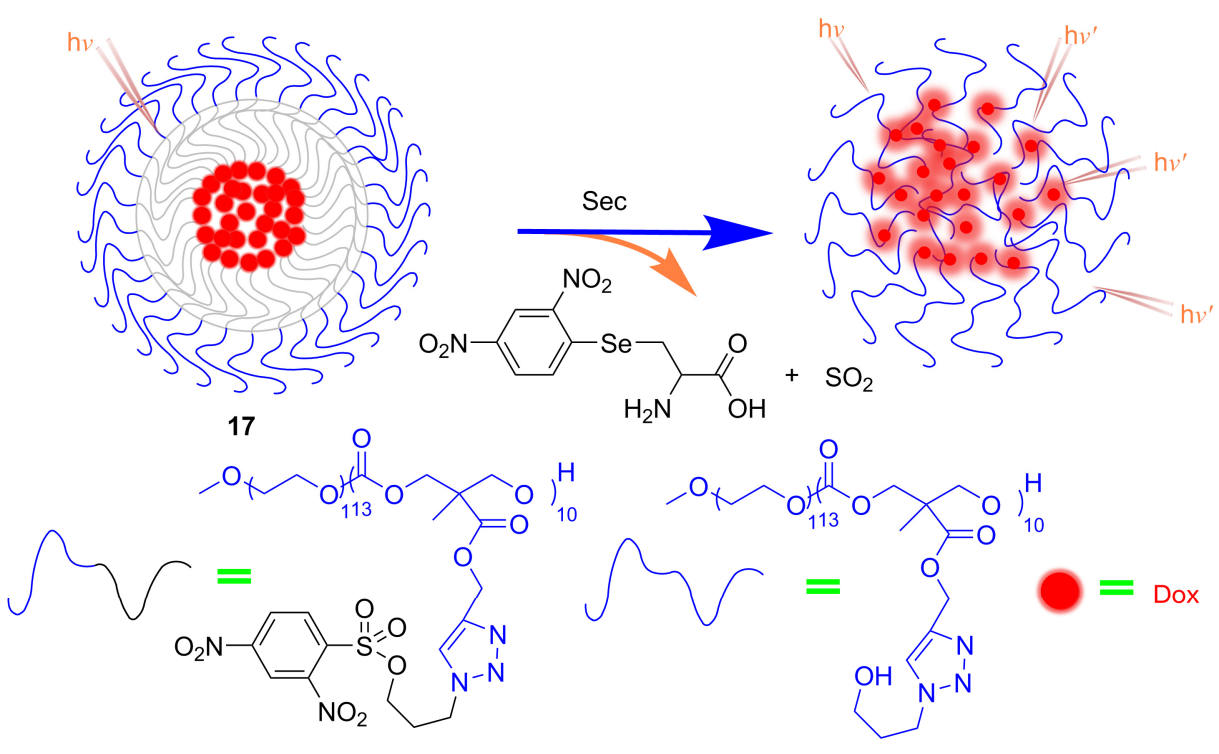

图 16 生物传感器 $\mathbf{1 7}$ 的结构和检测 $\operatorname{Sec}$ 的机理示意图

Figure 16 Structure of biosensor 17 and the mechanism of detecting Sec 
传感器以 $\mathrm{mAP}$ 作为碳源, 可通过简单的溶剂热法制备. 通过磺酰胺键将传感器 18 表面上的 2,4-二硝基苯磺酰 基(DNS)基团共价共轭，使 CD-DNS 作为硒醇具有很高 的敏感性和选择性. 该纳米传感器对硒醇的抗药性高于 生物硫醇和其他生物物种, 并且已被证明能够对活细胞 中的外源和内源硒醇进行成像. 这是第一例可以在生理 条件下运行的基于 $\mathrm{CDs}$ 的硒醇苂光传感器.

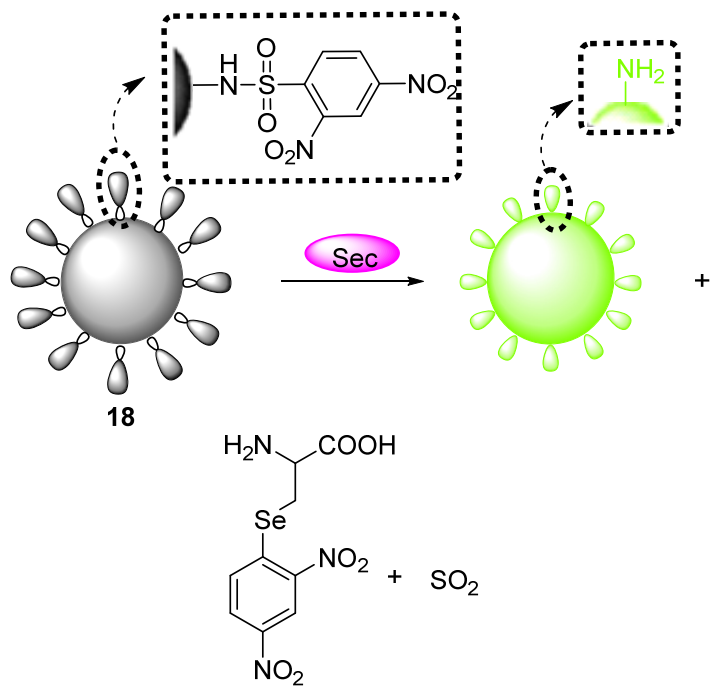

图 17 传感器 18 对 $\mathrm{Sec}$ 的识别机理图

Figure 17 Schematic illustration of the sensing mechanism of 18 for Sec

\section{2 硒化氢 $\left(\mathrm{H}_{2} \mathrm{Se}\right)$ 和硒醇荧光传感器}

硒化氢 $\left(\mathrm{H}_{2} \mathrm{Se}\right)$ 具有与 $\mathrm{H}_{2} \mathrm{~S}$ 相似的结构, 是一种高活 性硒物种. 通过谷胱甘肽( GSH) 和其它生物系统还原亚 硒酸盐而产生的膳食硒化合物的一种重要的硒代谢中 间产物, 其在生物体内含量与许多重要的生理和病理过 程有关 ${ }^{[43]}$. 因此, 开发一种具有高灵敏度和高选择性的 可用于检测 $\mathrm{H}_{2} \mathrm{Se}$ 的荧光传感器是非常有必要的.

硒醇是生命体内重要的活性物质, 对维持生命机体 正常运行具有重要的生理意义 ${ }^{[45]}$. 近年来, 随着高选择 性荧光有机分子传感器的不断被开发, 能够检测细胞内 硒醇分子的传感器被开发, 有望用于诊断与硒缺乏和过 量相关的人类疾病 ${ }^{[46]}$. 硫醇由于和硒醇具有相似的化 学性质, 从而成为硒醇苂光传感器设计的主要干扰. 迄
今为止，所报道的能够检测硒醇的荧光传感器还比较 少.

2016 年, 唐波等 ${ }^{[47]}$ 设计开发了一种用于检测 $\mathrm{H}_{2} \mathrm{Se}$ 的荧光传感器 19 , 其对 $\mathrm{H}_{2} \mathrm{Se}$ 表现出较高的敏感度和良 好的选择性，超过 $\mathrm{Sec}, \mathrm{H}_{2} \mathrm{~S}$, 活性氧(ROS)和其它含硫元 素衍生物(图 18). 该传感器被成功应用于活细胞和内源 性 $\mathrm{H}_{2} \mathrm{Se}$ 苂光成像研究. 除此之外, 该传感器也被应用于 $\mathrm{Na}_{2} \mathrm{SeO}_{3}$ 诱导的 $\mathrm{HepG} 2$ 细胞在细胞凋亡过程中 $\mathrm{H}_{2} \mathrm{Se}$ 含 量的检测. 研究发现, 与有氧条件下相比, 在缺氧条件 下观察到 $\mathrm{H}_{2} \mathrm{Se}$ 含量增加的同时 ROS 保持低水平. 该研 究结果表明, Se 元素对缺氧性肿瘤的抗癌机制是通过非 氧化应激产生的. 该结果为进一步研究 $\mathrm{H}_{2} \mathrm{Se}$ 和含 $\mathrm{Se}$ 化 合物在各种生理和病理环境中的生物学功能提供依据. 该工作的亮点是发现了硒对缺氧性实体瘤的抗癌机制 是通过非氧化应激.

2016 年, Rao 等 ${ }^{[48]}$ 报道了一种用于检测 $\mathrm{Cys}-\mathrm{SeH}$ 的 水溶性苂光传感器 20 (图 19). 其结构设计中, 巧妙地引 入了三唑连接吡喃官能团的葡萄糖基结构. 研究发现传 感器 20 在 Cys-SeH 的磷酸盐缓冲溶液(PBS)中反应释放 出苂光产物. 在 20 种其它氨基酸存在的情况下, 传感器 显示出约 210 倍的荧光增强. 其最小检测限为 $(1.5 \pm 0.2)$ $\times 10^{-7} \mathrm{~mol} / \mathrm{L}$. 为了进一步证明传感器对 Cys-SeH 的选 择性，他们将该结构扩展到了不同的其它硒化合物中， 对含有 2 个 $\mathrm{NO}_{2} 、 5$ 个氟、2 个氟、 1 个氟以及没有氟存 在的 5 个不同的衍生物进行了荧光性能研究，证实了对 硝基苯磺酰基在传感器中对 $\mathrm{Cys}-\mathrm{SeH}$ 有选择性识别作 用. 通过等温滴定量热法研究传感器在 PBS 缓冲液、牛 血清和硅胶表面对 Cys-SeH 选择性研究发现, 糖基引入 到传感器结构中, 大大提高了传感器的生物应用范围.

2017 年, 唐波课题组 ${ }^{[49]}$ 基于花菁结构设计合成了 一种具有特异性受体单元 1,2-二噻烷-4,5-二醇的 $\mathrm{H}_{2} \mathrm{Se}$ 荧光传感器 21, 其对 $\mathrm{H}_{2} \mathrm{Se}$ 的选择性高于 $\mathrm{RSH}, \mathrm{H}_{2} \mathrm{~S}$ 和 $\mathrm{Sec}$, 被成功应用于活细胞内 $\mathrm{H}_{2} \mathrm{Se}$ 的荧光成像研究(图 20). 该研究结果表明在缺氧条件下和用 $\mathrm{Na}_{2} \mathrm{SeO}_{3}$ 处理 后, $\mathrm{HepG} 2$ 细胞会产生 $\mathrm{H}_{2} \mathrm{Se}$, 且 $\mathrm{H}_{2} \mathrm{Se}$ 在缺氧条件下逐渐 被积累. 该研究结果表明 Se 对缺氧实体瘤的抗癌机制 是通过非氧化应激发生的. 该传感器有望被用于 $\mathrm{H}_{2} \mathrm{Se}$
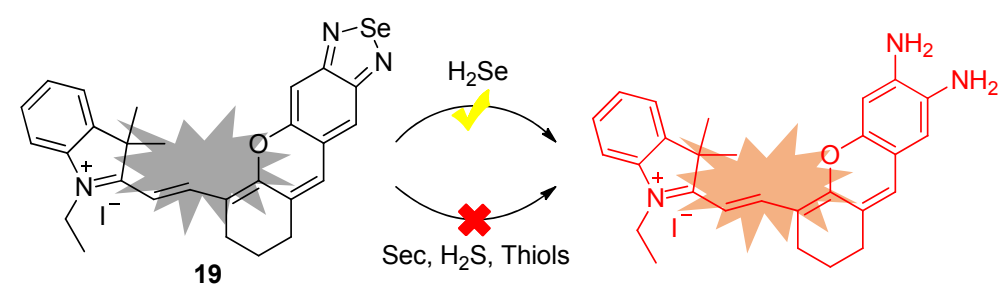

图 18 苂光传感器 19 的化学结构和对 $\mathrm{H}_{2} \mathrm{Se}$ 选择性反应机理

Figure 18 Chemical structure of fluorescent sensor 19 and the mechanism of selective reaction to $\mathrm{H}_{2} \mathrm{Se}$ 


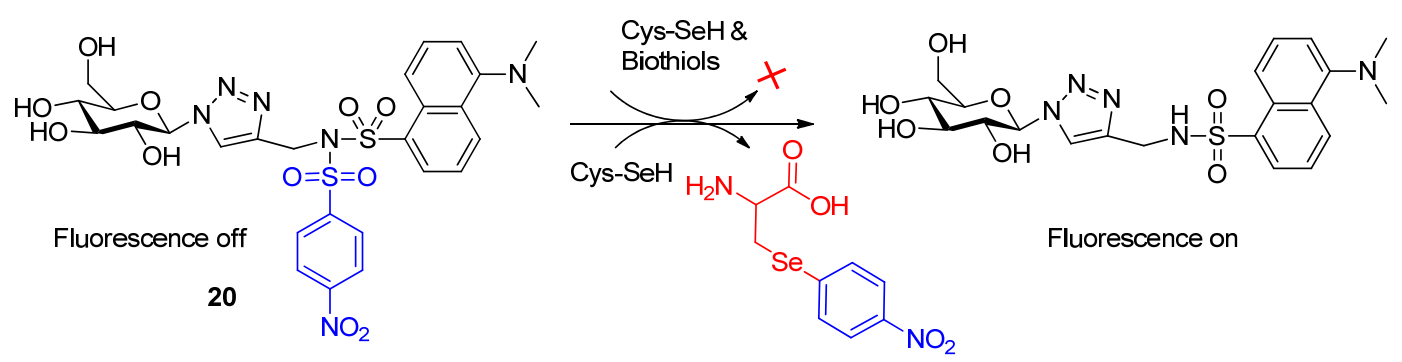

图 19 传感器 20 的化学结构和对硝基苯磺酰基在结构中对 $\mathrm{Cys}-\mathrm{SeH}$ 的选择性识别示意图

Figure 19 Chemical structure of sensor 20 and the scheme of selective recognition of Cys-SeH by $p$-nitrobenzenesulfonyl in structure

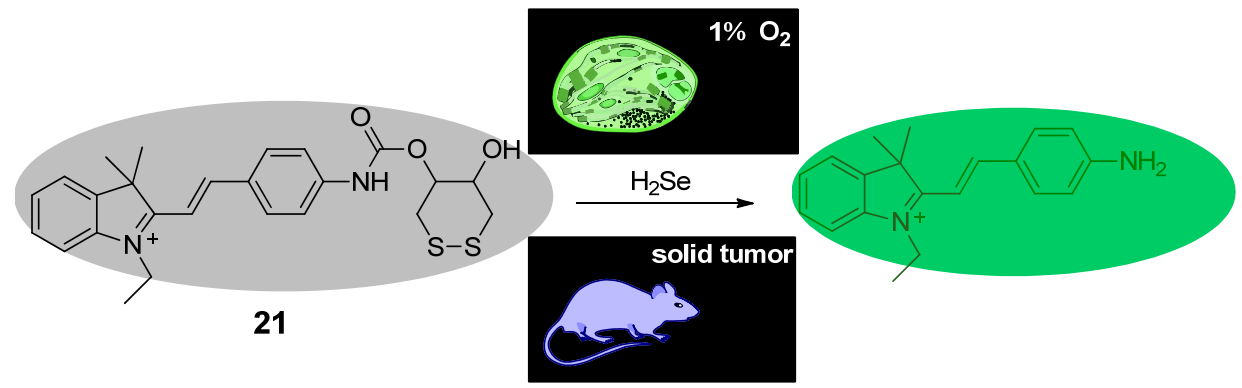

图 20 苂光传感器 21 的化学结构和活细胞内成像示意图

Figure 20 Chemical structure of fluorescent sensors 21 and the scheme of intracellular imaging

和 Se 元素的抗癌机制研究.

宋钦华等 ${ }^{[00]}$ 设计合成了两种关于硒醇的苂光传感 器 22 和 23 , 其结构是由两种氟硼二吡咯苂光团和苂光 猝灭基团 2,4-二硝基苯氧基组成(图 21). 两种传感器通 过芳香亲核取代反应对硒醇产生响应，从而释放荧光 团. 其检测机理归因于硒醇和硫醇 $\mathrm{p} K_{\mathrm{a}}$ 值的不同. 在生 理 $\mathrm{pH}$ 条件下, 相比较于硫醇, 传感器对硒醇和硒代半 胱氨酸具有更高的选择性. 传感器 $\mathbf{2 3}$ 相较于传感器 $\mathbf{2 2}$, 具有更高的灵敏性, 其检测限为 $9 \mathrm{nmol} / \mathrm{L}$, 而 22 的检测 限为 $16 \mathrm{nmol} / \mathrm{L}$. 细胞成像实验研究表明传感器 23 能够 检测活细胞中外生的 Sec 和 $\mathrm{Na}_{2} \mathrm{SeO}_{3}$ 诱导的 Sec. 此外, 传感器 23 可实现活体动物体内的硒醇荧光成像研究.

2019 年, 张小玲等 ${ }^{[51]}$ 提出了一种简明的设计策略, 利用分子内光致电子转移(PET)过程来切换荧光, 从而 获得 $\mathrm{H}_{2} \mathrm{Se}$ 的可激活荧光传感器 $\mathbf{2 4}$ (图 22). 该传感器能 够选择性地与 $\mathrm{H}_{2} \mathrm{Se}$ 反应, 不受细胞内反应物种的干扰, 并已被成功地用于溶酶体中 $\mathrm{H}_{2} \mathrm{Se}$ 含量成像检测. 此外, 在传感器 24 的辅助下, 该课题组证明了在低氧条件下, HepG2 细胞中可以产生并逐渐积累溶酶体 $\mathrm{H}_{2} \mathrm{Se}$. 这些 应用使该结构成为在生物学和病理学中破译 $\mathrm{H}_{2} \mathrm{Se}$ 对溶 酶体生物学效应的潜在新候选者.

为了研究亚硒酸盐诱导实体瘤细胞凋亡是否可以 归因于氧化应激. 基于硒醇对 $\mathrm{Au}-\mathrm{S}$ 键的快速取代反 应, 2016 年 Tang 等 ${ }^{[52]}$ 设计合成了硒醇纳米探针 25 (图 23). 该探针与 $\mathrm{QCy} 7-\mathrm{H}_{2} \mathrm{O}_{2}$ 分子探针一起, 能够监测活 细胞和活体中硒醇及 $\mathrm{H}_{2} \mathrm{O}_{2}$ 的浓度. 观察到在常氧条件

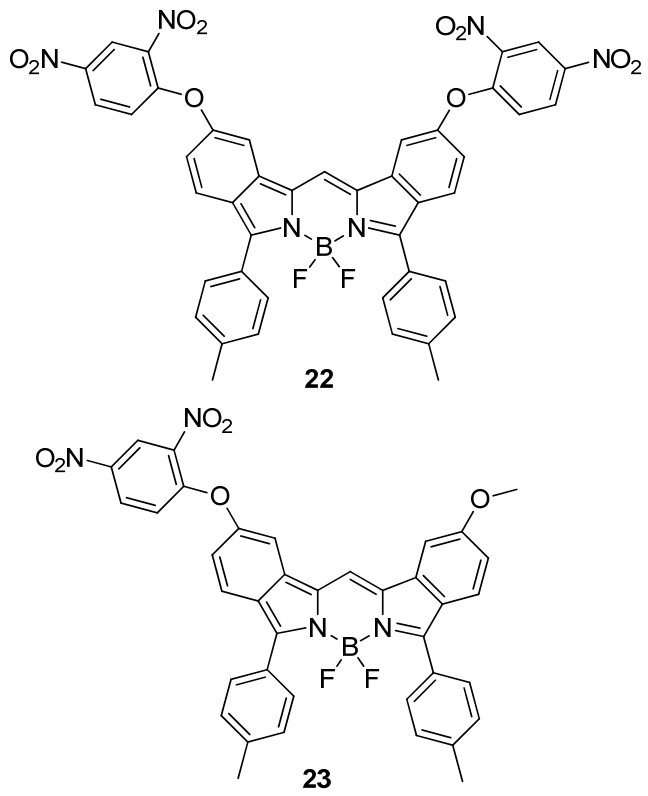

图 21 荧光传感器 22 和 23 的化学结构

Figure 21 Chemical structures of fluorescent sensors 22 and $\mathbf{2 3}$

下，硒醇含量缓慢增加，并且 $\mathrm{H}_{2} \mathrm{O}_{2}$ 水平快速升高. 然而， 在低氧条件下, 硒醇浓度急剧增加, $\mathrm{H}_{2} \mathrm{O}_{2}$ 含量几乎没有 波动. 根据上述结果，可以预测亚硒酸盐诱导的肿瘤细 胞调亡归因于非氧化应激. 该研究成果为研究亚硒酸钠 诱导肿瘤细胞调亡的机制提供了有力的工具.

\section{$3 \mathrm{Se}$ (IV)荧光传感器}

硒在无机形式时, 有四种化合价态, 包括元素(Se)、 


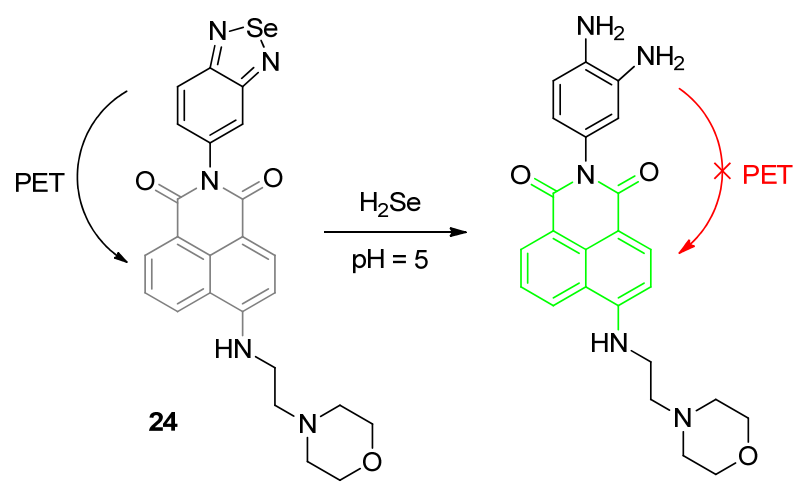

图 22 荧光传感器 $\mathbf{2 4}$ 被 $\mathrm{H}_{2} \mathrm{Se}$ 激活的机理过程

Figure 22 Proposed mechanism for $\mathrm{H}_{2} \mathrm{Se}$ activation of chemsensor 24
硒化物 $\left(\mathrm{Se}^{2-}\right)$ 、亚硒酸盐 $\left[\mathrm{SeO}_{3}^{2-}, \mathrm{Se}(\mathrm{IV})\right]$ 和硒酸盐 $\left[\mathrm{SeO}_{4}^{2-}, \mathrm{Se}(\mathrm{VI})\right]$. $\mathrm{Se}(\mathrm{IV})$ 和 $\mathrm{Se}(\mathrm{VI})$ 均具有典型的毒性. 毒 理学实验表明, $\mathrm{Se}(\mathrm{IV})$ 的毒性比 $\mathrm{Se}(\mathrm{VI})$ 高. $\mathrm{SeO}_{3}^{2-}$ $[\mathrm{Se}(\mathrm{IV})]$ 荧光探针原理主要涉及配位作用、与二胺基团 反应的策略以及与纳米结构的相互作用 ${ }^{[45]} . \mathrm{Se}(\mathrm{IV})$ 与包 含 1,2 -二胺基团的物质反应形成吡唑硒醇.

纳米荧光传感器具有响应速度快、选择性好、灵敏 度和稳定性及生物相容性好等优点 ${ }^{[53-54]}$, 唐波等 ${ }^{[55]}$ 根据 硒能特异性破坏 $\mathrm{Au}-\mathrm{S}$ 键并在金纳米颗粒(AuNPs)表面 形成更稳定的 $\mathrm{Au}-\mathrm{Se}$ 键的原理, 设计开发了一种用于 检测硒醇的近红外苂光纳米传感器 26(图 24). 该传感器 可以用于 $\mathrm{Na}_{2} \mathrm{SeO}_{3}$ 诱导的 $\mathrm{HepG} 2$ 细胞调亡过程中硒醇

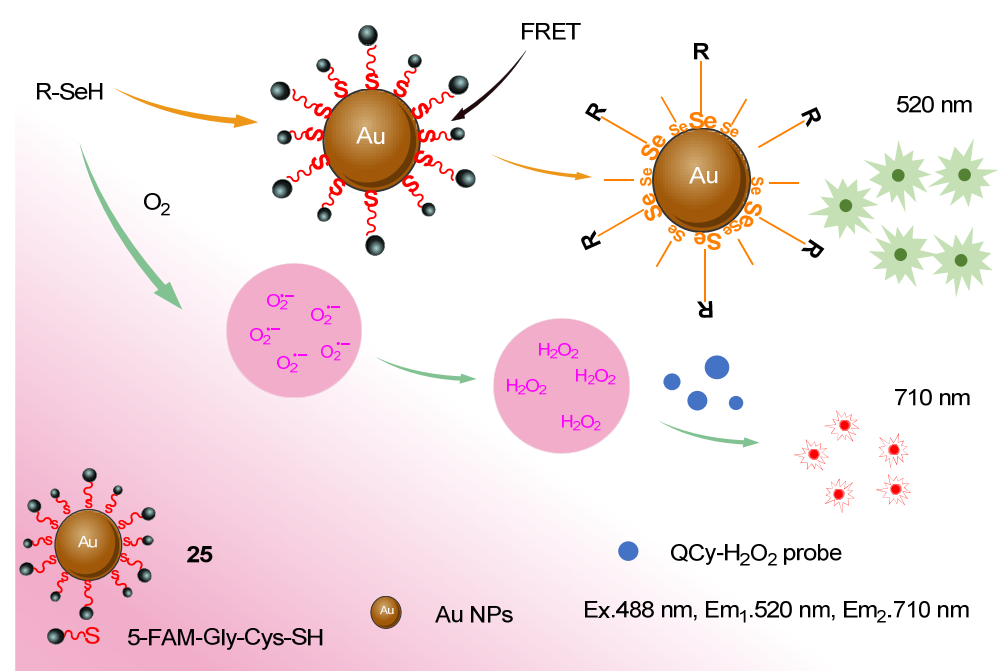

图 23 传感器 25 的设计策略及对硒醇的苂光成像

Figure 23 Design strategy and fluorescence imaging for selenol of chemsensoor 25

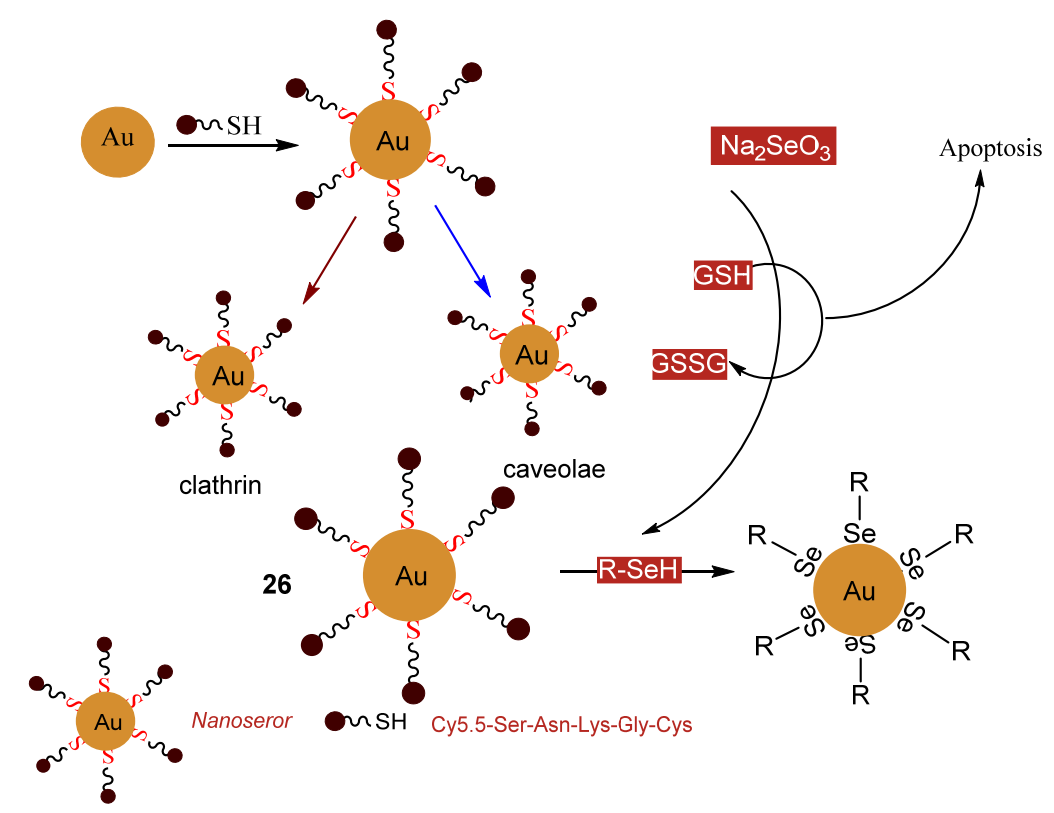

图 24 纳米传感器 $\mathbf{2 6}$ 检测 $\mathrm{Na}_{2} \mathrm{SeO}_{3}$ 诱导的癌细胞中硒醇的原理图

Figure 24 Schematic illustration of the nanosensor $\mathbf{2 6}$ for the detection of selenol in cancer cells induced by $\mathrm{Na}_{2} \mathrm{SeO}_{3}$ 
含量变化的荧光成像监测. 在低氧条件下, $\mathrm{Na}_{2} \mathrm{SeO}_{3}$ 诱 导的肿瘤细胞调亡与硒醇水平密切相关. 该结构可以作 为监测硒醇的有力工具, 并可以在多种生理和病理条件 下探索硒醇的生理功能. 该纳米传感器的设计策略为硒 化学的相关研究提供了一种新的平台.

Zhao 等 ${ }^{[56]}$ 报道了一种选择性检测硒(IV)的纳米苂 光传感器 27(图 25). 该纳米传感器可以通过靶向诱导信 号原位检测 $\mathrm{Se}(\mathrm{IV})$. 该传感器由二氧化硅纳米粒子核和 含有 $\operatorname{Se}(\mathrm{IV})$ 诱导的荧光分子 3,3'-二氨基联苯胺(DAB)涂 层组成. 在没有 $\mathrm{Se}(\mathrm{IV})$ 存在时, 纳米传感器没有苂光发 射信号. 但当纳米传感器与 $\operatorname{Se}(\mathrm{IV})$ 靶向结合时, 纳米传 感器被激活, 其机理为 $\operatorname{Se}(\mathrm{IV})$ 首先在纳米传感器的一个 小区域上富集, 然后在 $420 \mathrm{~nm}$ 激发波长激发时, 纳米传 感器在 $530 \mathrm{~nm}$ 发射波长处产生荧光发射信号. 其苂光 强度与 $\mathrm{Se}(\mathrm{IV})$ 浓度成正比, 可以通过荧光显微镜观察纳 米传感器富集过程. 该策略是第一次实现 $\mathrm{Se}(\mathrm{IV})$ 在纳米 传感器上富集. 该研究结果为在生物体系中如何设计原 位靶向的荧光纳米传感器提供思路.

田熙科等 ${ }^{[57]}$ 开发了一种比率型苂光纳米传感器 $\mathbf{2 8}$, 该传感器通过将识别分子 3,3'-二氨基联苯胺(DAB)连接 到羧基修饰的 $\mathrm{CdTe} @ \mathrm{SiO}_{2}$ 表面用于精确原位检测 $\mathrm{SeO}_{3}^{2-}$ (图 26). 研究发现, $\mathrm{DAB}$ 和 $\mathrm{SeO}_{3}^{2-}$ 之间发生表面 螯合反应, 可以选择性、有效地增强 $\mathrm{DAB}$ 在二氧化硅 纳米球表面的荧光强度. 在 $\mathrm{SeO}_{3}^{2-}$ 存在的情况下, 该纳 米传感器在单一激发波长下显示出 Se-DAB 和 CdTe 量
子点的两种特征荧光发射信号. 通过对 $\mathrm{SeO}_{3}^{2-}$ 的选择性 篮选研究发现, $F_{530} / F_{635}$ 的线性比率在 $0 \sim 2.5 \mu \mathrm{mol} / \mathrm{L}$ 范 围内随 $\mathrm{SeO}_{3}^{2-}$ 浓度的增加而增加, 其检出限低至 6.68 $\mathrm{nmol} / \mathrm{L}(0.53 \mu \mathrm{g} / \mathrm{L})$. 该研究结果所报道的纳米传感器具 有在水溶液体系中进行原位监测的能力, 无需任何分离 步骤. 利用该传感器测定富硒食品中硒的含量, 其检测 结果与电感耦合等离子体原子发射光谱法(ICP-AES)测 定结果一致.

2016 年, 张勇等 ${ }^{[58]}$ 成功制备了一种简单、快速和灵 敏检测亚硒酸盐的室温磷光传感器 29(图 27). 当检测体 系中存在谷胱甘肽时, 金属 $\mathrm{Mn}$ 掺杂的 $\mathrm{ZnS}$ 量子点的室 温磷光被淬灭. $\mathrm{Mn}-\mathrm{ZnS}$ 量子点的磷光淬灭归因于亚硒 酸盐和谷胱甘肽反应产生的 $\mathrm{HSe}^{-}$. 在实验条件下, 其 线性范围为 $0.1 \sim 5.0 \mu \mathrm{mol} \cdot \mathrm{L}^{-1}$, 检测限为 $0.085 \mu \mathrm{mol} \cdot$ $\mathrm{L}^{-1}$. 该传感器被成功应用于亚硒酸钠片、亚硒酸钠及维 生素 $\mathrm{E}$ 注射液中亚硒酸盐含量的测定. 该研究方法可以 测定出从微摩尔甚至更低水平的硒化物浓度. 该水平接 近于各种细菌和哺乳动物细胞生长所需的最佳硒化物 浓度.

\section{4 结论与展望}

硒元素作为哺乳动物成长最为重要的微量元素之 一，目前被认为是一种能够抗病毒的营养素，可以提高 肌体的免疫性能, 预防癌症和心血管疾病等. 本文总结 了含硒物质如 Sec、 $\mathrm{H}_{2} \mathrm{Se}$ 和 $\mathrm{Se}(\mathrm{IV})$ 苂光传感器方面的研

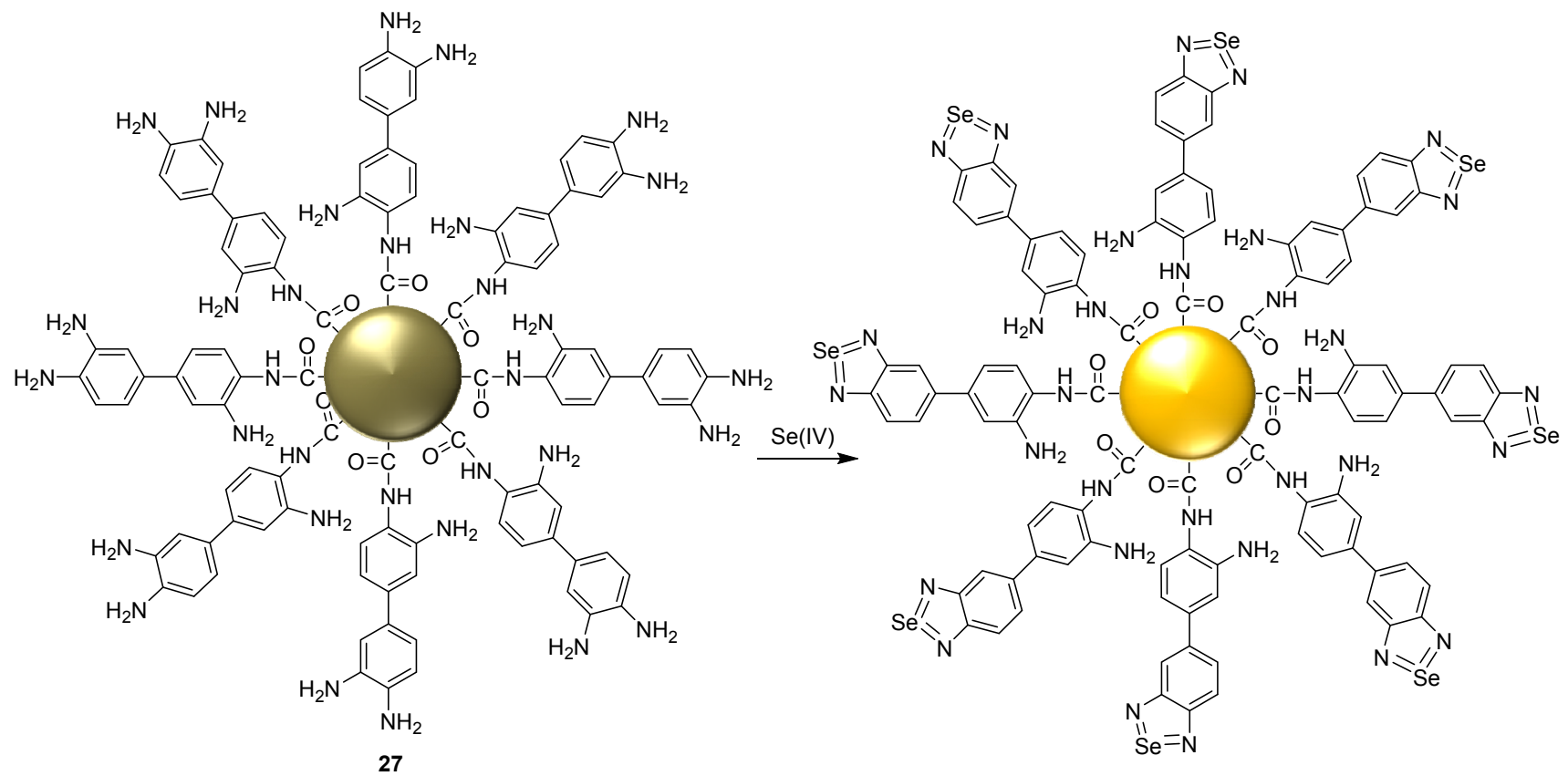

图 25 纳米苂光传感器 $\mathbf{2 7}$ 的化学结构和 $\mathrm{Se}(\mathrm{IV})$ 存在时纳米传感器开启苂光信号的示意图

Figure 25 Chemical structure of nanosensor 27 and schematic of the nanosensors turning on fluorescence signal in the presence of $\mathrm{Se}(\mathrm{IV})$ 


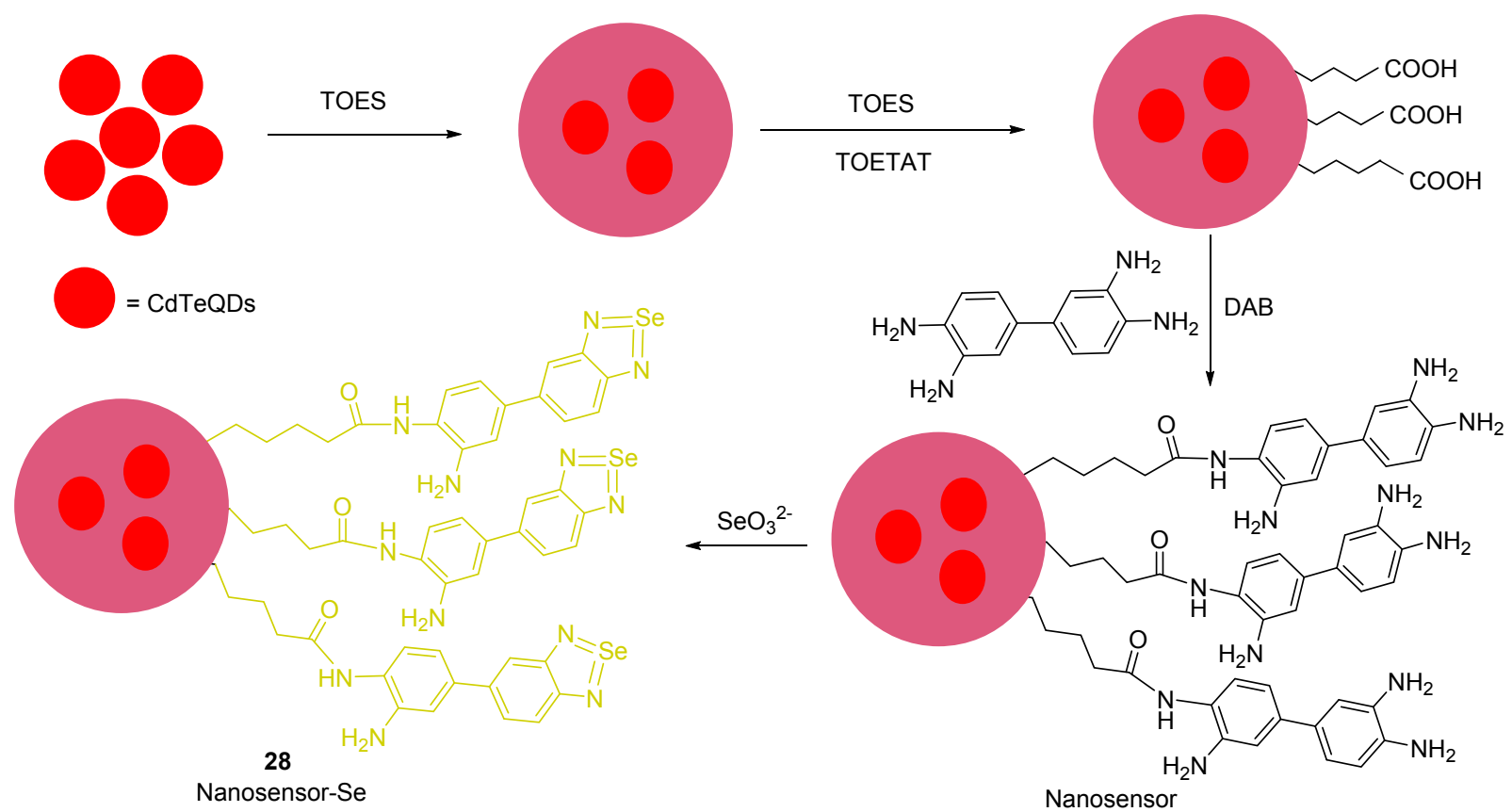

图 26 比率型苂光纳米传感器 28 的制备原理和 $\mathrm{SeO}_{3}^{2-}$ 的传感示意图

Figure 26 Preparation principle of ratio fluorescence nanosensor 28 and the schematic diagram of sensing principle of $\mathrm{SeO}_{3}^{2-}$

究进展. 近年来, 尽管在含硒元素物质苂光传感器设计 和检测及生物成像方面得到了飞速发展, 但依旧存在一 些问题和挑战. 由于含硒物质参与的高选择性反应的数 量有限, 所以硒化物传感器种类仍然有限. 在设计硒化 物的生物体系苂光传感器时需要考虑选择性、灵敏性、 光稳定性、低细胞毒性、低自氧化性、良好的水溶性、 细胞渗透性和生物环境中物质的低干扰等性能. 希望本 文的总结能够对含硒物质荧光传感器的设计提供帮助.

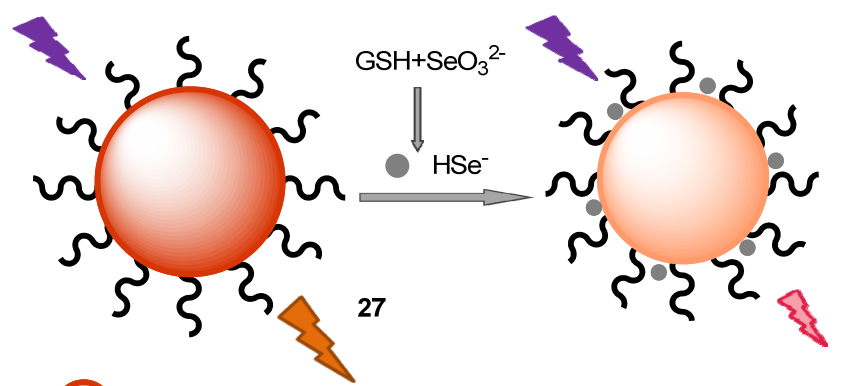

Mn-ZnS QDs

$\mathrm{HSe}^{-}$ก $\mathrm{MPA}$

图 $27 \mathrm{HSe}^{-}$引起的传感器 29 的室温磷光猝灭检测亚硒酸盐 示意图

Figure 27 Schematic diagram of room temperature phosphorescence quenching of sensor 29 induced by $\mathrm{HSe}^{-}$for detection of selenite

\section{References}

[1] Oldfield, J. E. New Zeal. Vet. J. 1974, 22, 85.

[2] Schwarz, K.; Foltz, C. M. J. Am. Chem. Soc. 1957, 79, 3292.

[3] Ogawa-Wong, A. N.; Berry, M. J.; Seale, L. A. Nutrients 2016, 8, 80.
[4] Rayman, M. P. The Lancet 2000, 356, 233.

[5] Rayman, P. M. The Lancet 2012, 379, 1256.

[6] Du, X.; Wang, C.; Liu, Q. Curr. Top. Med. Chem. 2016, 16, 835.

[7] Bhabak, K. P.; Mugesh, G. Acc. Chem. Res. 2010, 43, 1408.

[8] Lou, Z.; Li, P.; Han, K. Acc. Chem. Res. 2015, 48, 1358.

[9] Manjare S. T.; Kim, Y.; Churchill, D. G. Acc. Chem. Res. 2014, 47, 2985.

[10] Reich, H. J.; Hondal, R. J. ACS Chem. Biol. 2016, 11, 821.

[11] Zhang, J.; Zhang, J.; Yan, Z.; Xie, J. Chin. J. Org. Chem. 2019, 39, 3051 (in Chinese). (张继东, 张俊, 严瞻, 谢娟平, 有机化学, 2019, 39, 3051.)

[12] Yang, M.; Su, N.; Li, Y.; Wang, L.; Ma, L.; Zhang, Y.; Li, J.; Yang, B.; Kang, L. Chin. J. Org. Chem. 2018, 38, 636 (in Chinese). (杨美盼, 苏娜, 李玉香, 王莉, 马利锋, 张媛, 李靖, 杨秉勤, 康龙丽, 有机化学, 2018, 38, 636.)

[13] Liu, H.-W.; Chen, L.; Xu, C.; Li, Z.; Zhang, H.; Zhang, X.-B.; Tan, W. Chem. Rev. 2015, 115, 7840.

[14] Gao, M.; Yu, F.; Lv, C.; Choo, J.; Chen, L. Chem. Soc. Rev. 2017, 46, 2237.

[15] Kwok, R. T. K.; Leung, C. W. T.; Lam, J. W. Y.; Tang, B. Z. Chem. Soc. Rev. 2015, 44, 4228.

[16] Li, J.; Chen, L.; Du, L.; Li, M. Chem. Soc. Rev. 2013, 42, 662.

[17] Xu, Z.; Kim, S. K.; Yoon, J. Chem. Soc. Rev. 2010, 39, 1457.

[18] Yue, Y.; Huo, F.; Cheng, F.; Zhu, X.; Mafireyi, T.; Strongin, R. M.; Yin, C. Chem. Soc. Rev. 2019, 48, 4155.

[19] Weekley, C. M.; Harris, H. H. Chem. Soc. Rev. 2013, 42, 8870.

[20] Maeda, H.; Katayama, K.; Matsuno, H.; Uno, T. Angew. Chem., Int. Ed. 2006, 45, 1810.

[21] Cheng, D.; Pan, Y.; Yin, B.-C.; Yuan, L.; Zhang, X. B. Chin. Chem. Lett. 2017, 28, 1987.

[22] Zhang, B.; Ge, C.; Yao, J.; Liu, Y.; Xie, H.; Fang, J. J. Am. Chem. Soc. 2015, 137, 757.

[23] Zhang, H.; Li, M.; Feng, W.; Feng, G. Dyes Pigm. 2018, 149, 475.

[24] Sun, Q.; Yang, S.-H.; Wu, L.; Dong, Q.-J.; Yang, W.-C.; Yang, G.-F. Anal. Chem. 2016, 88, 6084.

[25] Li, J.; Zhang, C.-F.; Yang, S. -H.; Yang, W. C.; Yang, G.-F. Anal. Chem. 2014, 86, 3037.

[26] Feng, W.; Li, M.; Sun, Y.; Feng, G. Anal. Chem. 2017, 89, 6106.

[27] Li, M.; Feng, W.; Zhai, Q.; Feng, G. Biosens. Bioelectron. 2017, 87, 894. 
[28] Zhang, P.; Ding, Y.; Liu, W.; Niu, G.; Zhang, H.; Ge, J.; Wu, J.; Li, Y.; Wang, P. Sens. Actuators, B 2018, 264, 234.

[29] Tian, Y.; Xin, F.; Gao, C.; Jing, J.; Zhang, X. J. Mater. Chem. B 2017, 5, 6890 .

[30] Kong, F.; Hu, B.; Gao, Y.; Xu, K.; Pan, X.; Huang, F.; Zheng, Q.; Chen, H.; Tang, B. Chem. Commun. 2015, 51, 3102.

[31] Han, X.; Wang, R.; Song, X.; Yu, F.; Chen, L. Anal. Chem. 2018, 90, 8108.

[32] Luo, X. Z.; Wang, R.; Lv, C. Z.; Chen, G.; You, J. M.; Yu, F. B. Anal. Chem. 2020, 92, 1589.

[33] Han, X. Y.; Song, X. Y.; Yu, F. B.; Chen, L. X. Adv. Funct. Mater. 2017, 1700761.

[34] Zhang, S.; Wang, Q.; Liu, X.; Zhang, J.; Yang, X.-F.; Li, Z.; Li, H. Anal. Chem. 2018, 90, 4119.

[35] Chen; H.; Dong, B.; Tang, Y.; Lin, W. Chem.-Eur. J. 2015, 21, 11696.

[36] Zhang, L.; Kai, X.; Zhang, Y.; Zheng, Y.; Xue, Y.; Yin, X.; Zhao, J. Analyst 2018, 143, 4860.

[37] Zhang, L.; Shi, Y.; Sheng, Z.; Zhang, Y.; Kai, X.; Li, M.; Yin, X. ACS Sens. 2019, 4, 3147.

[38] Wang, C.; Chen, Q.; Wang, Z.; Zhang, X. Angew. Chem., Int. Ed. 2010, 49, 8794.

[39] Cheng, Y.; He, C.; Xiao, C.; Ding, J.; Ren, K.; Yu, S.; Zhuang, X.; Chen, X. Polym. Chem. 2013, 4, 3851.

[40] Du, J.-Z.; Du, X.-J.; Mao, C.-Q.; Wang, J. J. Am. Chem. Soc. 2011, 133, 17560 .

[41] Yan, Q.; Zhao, Y. J. Am. Chem. Soc. 2013, 135, 16300.

[42] Nan Y.; Zhao, W.; Xu, X.; Au, C.-T.; Qiu, R. RSC Adv. 2015, 5, 69299.

[43] Wang, Q.; Zhang, S. G.; Zhong, Y. G.; Yang, X. F.; Li, Z.; Li, H.
Anal. Chem. 2017, 89, 1734.

[44] Weekley. C.; Aitken, J.; Vogt, S.; Finney, L.; Paterson, D.; Jonge, M.; Howard, D.; Witting, P.; Musgrave, I.; Harris, H. J. Am. Chem. Soc. 2011, 133, 18272.

[45] Reich, H. J.; Hondal, R. J. ACS Chem. Biol. 2016, 11, 821.

[46] Wu, D.; Chen, L.; Kwon, N.; Yoon, J. Y. Chem 2016, 674.

[47] Kong, F.; Ge, L.; Pan, X.; Xu, K.; Liu, X.; Tang, B. Chem. Sci. 2016, 7, 1051.

[48] Areti, S.; Verma, S. K.; Bellare, J.; Rao, C. P. Anal. Chem. 2016 88,7259 .

[49] Kong, F.; Zhao, Y.; Liang, Z.; Liu, X.; Pan, X.; Luan, D.; Xu, K.; Tang, B. Anal. Chem. 2017, 89, 688.

[50] Dai, C.-G.; Wang, J. L.; Song, Q.-H. J. Mater. Chem. B 2016, 4, 6726.

[51] Tian, Y.; Xin, F. Y.; Jing, J.; Zhang, X. L. J. Mater. Chem. B 2019, 7, 2829.

[52] Liu, X. J.; Hu, B.; Cheng, R.-R.; Kong, F. P.; Pan, X. H.; Xu, K. H.; Tang, B. Chem. Commun. 2016, 52, 6693.

[53] Dreaden, E. C.; Alkilany, A. M.; Huang, X.; Murphy, C. J.; El-Sayed, M. A. Chem. Soc. Rev. 2012, 41, 2740

[54] Resch-Genger, U.; Grabolle, M.; Cavaliere-Jaricot, S.; Nitschke, R.; Nann, T. Nat. Methods 2008, 5, 763.

[55] Hu, B.; Cheng, R.; Liu, X.; Pan, X.; Kong, F.; Gao, W.; Xu, K.; Tang, B. Biomaterials 2016, 92, 81 .

[56] Liang, S.; Chen, J.; Pierce, D. T.; Zhao, J. X. ACS Appl. Mater. Interfaces 2013, 5, 5165 .

[57] Chen, L.; Tian, X.; Zhao, Y.; Li, Y.; Yang, C.; Zhou, Z.; Liu, X. Analyst 2016, 141, 4685.

[58] Chen, J.; Zhu, Y.; Zhang, Y. RSC Adv. 2016, 6, 62193.

(Cheng, F.) 